\title{
Local Mean Decomposition Combined with SVD and Application in Telemetry Vibration Signal Processing
}

\author{
Zhenxing LI \\ Unit 94 \\ PLA 91550 \\ Dalian, China \\ lizhenxing@126.com
}

\author{
Weixiao DAI \\ Unit 94 \\ PLA 91550 \\ Dalian, China \\ 2003slsl@163.com
}

\begin{abstract}
To suppress the noise effect on the performance of local mean decomposition (LMD) in signal processing, a new method combined with singular value decomposition (SVD) denosing was proposed in this work. SVD is applied to denoise the observed signal, and then the signal decompose to series product functions (PF) by LMD, meanwhile, SVD is applied to denoise the PF and sum the PF to LMD again. This method can suppress the noise effect to attain high precision PF for time-frequency analysis. Simulation signal and telemetry vibration signal processing results show the effectiveness of the method.
\end{abstract}

Keywords-local mean decomposition; SVD; telemetry; vibration signal processing; denoising

\section{INTRODUCTION}

Non-stationary signal widely exists in a variety of engineering applications, such as fault detection [1], medical signal processing and speech signal processing, and so on. LMD is similar to experiment mode decomposition (EMD), its essence is decompose the signal to series PFs, based on this, we can attain instantaneous frequency and instantaneous energy. So far, LMD method has achieved more applications in engineering. In the actual, acquisition signals are inevitably subjected to noise interference, so signal envelope extraction would be inaccurate, thus affecting the signal for further time frequency processing. Based on this point, this paper combines SVD and LMD method, using SVD to signal denoising process, then use LMD for decomposition, to further protect the LMD extraction of envelope precision and accuracy of pure FM signal, the PFs denoise by SVD and sum to LMD again, then we can obtain more accurate signal envelope and pure FM signal for further time frequency analysis. Simulation signal and telemetry vibration signal processing results show the effectiveness of this method.

\section{PRINCIPLE OF LMD}

Unlike Fourier transform and wavelet transform, LMD does not need a fixed basis function for signal decomposition, and then it is an adaptive signal decomposition method. The target of LMD is decomposing a signal to a series of PF, every PF corresponding to a product of an envelope signal and a pure FM signal. similar to EMD [2], in order to obtain the good time frequency distribution results, meanwhile, let the corresponding instantaneous frequency reflect the actual meaning, for multi-component signal, decompose it into single component signal can obtain more accurate instantaneous frequency and instantaneous energy results. The implement step of LMD can be summarized as follows [3]

Step1: Determine all the local extreme points of the signal $x(t)$, including the maxima and minima $n_{11}\left(k_{l}\right)$ $(l=1,2, \cdots, M)$, and local amplitude function $m_{11}(t)$ and local mean function $a_{11}(t)$ can compute by Eq.1 and Eq.2.

$$
\begin{aligned}
& m_{11}(t)=\frac{n_{11}\left(k_{l}\right)+n_{11}\left(k_{l+1}\right)}{2} \\
& a_{11}(t)=\frac{\left|n_{11}\left(k_{l}\right)-n_{11}\left(k_{l+1}\right)\right|}{2}
\end{aligned}
$$

where $k_{l}=k_{1}, k_{2}, \cdots, k_{M-1}, t \in\left[k_{l}, k_{l+1}\right]$.

Step2: Adopt the sliding average for all the local mean function and local amplitude function for smoothing. Then subtract the smoothing local mean function $\tilde{m}_{11}(t)$ from the original signal $x(t)$, and we get the separation signal $h_{11}(t)$, normalize $h_{11}(t)$ by smooth local amplitude function $\tilde{a}_{11}(t)$ as Eq.4.

$$
\begin{gathered}
h_{11}(t)=x(t)-\tilde{m}_{11}(t) \\
s_{11}(t)=\frac{h_{11}(t)}{\tilde{a}_{11}(t)}
\end{gathered}
$$

Step3: If $s_{11}(t)$ is not a pure FM signal in $[-1,1]$, take $s_{11}(t)$ as the original signal and repeat step1 to step2 till the separation signal meet the condition. Instantaneous phase and instantaneous frequency can be determined by Eq.5 and Eq.6 respectively.

$$
\begin{aligned}
\varphi_{1}(t) & =\arccos \left(s_{11}(t)\right) \\
f_{1}(t) & =\frac{d \varphi_{1}(t)}{2 \pi d t}
\end{aligned}
$$

Step4: Use all the local amplitude function product to obtain instantaneous amplitude expressed as Eq.7. 


$$
a_{1}(t)=a_{11}(t) a_{12}(t) \cdots a_{1 n}(t)=\prod_{q=1}^{n} a_{1 q}(t)
$$

And the first PF can be computed by Eq.8.

$$
P F_{1}(t)=a_{1}(t) s_{1 n}(t)
$$

Step5: Here separate the first PF from the original signal as Eq.9.

$$
u_{1}(t)=x(t)-P F_{1}(t)
$$

Then we let $u_{1}(t)$ as a new original signal and repeat step1 to step4 till all $u_{k}(t)$ meet the monotone function condition. Thus, the original signal can be expressed as the sum of PF components and a monotonic component [4].

$$
x(t)=\sum_{p=1}^{k} P F_{p}(t)+u_{k}(t)
$$

Under the noise interference condition, the amplitude of envelope signal extracted by LMD is uncertain in the range.

\section{SVD DENOISING}

In all kinds of denoising methods, SVD is a robust method in many cases [5]. SVD is a matrix decomposition method, let $A$ is a matrix with dimension $N \times M$. The target of SVD is to find two unitary matrixes $U \in R^{N \times N}$ and $V \in R^{M \times M}$ which meet the conditions $U U^{T}=I$ and $V V^{T}=I$ to decompose $A$. Let

$$
\Lambda=\left[\operatorname{diag}\left\{\lambda_{1}, \lambda_{2}, \cdots, \lambda_{p}\right\}\right]
$$

where $p=\min (N, M)$. Thus SVD can be expressed as Eq.12.

$$
A=U \Lambda V^{T}
$$

where $U$ and $V$ are orthogonal matrix with dimension $N \times N$ and $M \times M$ respectively. The diagonal elements in $\Lambda$ is singular value and array according to degressive order. For $\Lambda$ is a diagonal matrix, SVD can decompose a $N \times M$ dimension matrix with rank $K$ to sum of $K$ $N \times M$ dimension sub-matrix with rank 1 , where each submatrix can be obtained by two eigenvectors product by a weight as Eq.13.

$$
A=U \Lambda V^{T}=\sum_{i=1}^{K} u_{i} \lambda_{i} v_{i}^{T}=\sum_{i=1}^{K} \lambda_{i} A_{i}
$$

where $u_{i}$ and $v_{i}$ is the vector of i-th column of $U$ and $V$ respectively. $\lambda_{i}$ is the i-th singular value under degressive order. Thus, matrix can decomposition by SVD to a series sub-matrix $A_{i}$ product by the corresponding $\lambda_{i}$.

Using SVD to a vector need phase space reconstruction to obtain a matrix [6]. A one-dimensional signal can be constructed many kinds of matrix, such as Topeliz matrix, Cycle matrix and Hankel matrix. The matrix construct form influences the performance of SVD in the signal processing. Practice has proved that Hankel matrix can attain better performance for denoising. Let the observation signal as a vector $X=[x(1), x(2), \cdots, x(N)]$, then the Hankel matrix for $X$ can be expressed as follow.

$$
A=\left[\begin{array}{cccc}
x(1) & x(2) & \cdots & x(n) \\
x(2) & x(3) & \cdots & x(n+1) \\
\vdots & \vdots & \vdots & \vdots \\
x(N-n+1) & x(N-n+2) & \cdots & x(N)
\end{array}\right]
$$

Sometimes $A$ is called reconstruction phase space [7], where $1<n<N$. Let $m=N-n+1$, then $A \in R^{m \times n}$. If the observation signal in the presence of noise or mutation information, then $A$ can be written as Eq.15.

$$
A=A_{m}+W
$$

where $W$ corresponds to the noise in the signal and $W$ can be regarded as a perturbation in the matrix, $A_{m}$ corresponds to the smoothing signal portion in the reconstructed phase space trajectory of a matrix. Under the condition of $A$ is known, $A_{m}$ and $W$ are unknown, we can use the singular value to get the characterize, and further to obtain the approximated $A_{m}$ eliminating $W$, the implement the target of denoising. Research shows that, the smooth signal corresponding to the phase space attractor matrix is singular, noise or fault signaling mutation information constitutes the path matrix $W$ must be full rank matrix. Let the rank of $A_{m}$ is $k(k<n)$, then keep the first former $k$ singular value and set the others to 0 .

Another question of using SVD denoising is how to choose the singular value [8]; here we choose the increment of singular entropy criterion for singular value. At first, define singular spectrum is

$$
\sigma_{i}=\log \left(\frac{\lambda_{i}}{\sum_{j=1}^{n} \lambda_{i}}\right) \quad(i \leq n)
$$


Then $\sigma_{i}(i=1,2, \cdots, n)$ is the singular spectrum of $A$ by SVD. To get the signal information content with singular spectrum order changes, further define singular entropy as Eq.17.

$$
E_{k}=\sum_{i=1}^{k} \Delta E_{i} \quad(k \leq n)
$$

where $k$ is the rank of singular entropy and $\Delta E_{i}$ is the increment at the ${ }^{i}$-th, then the increment of singular entropy can be computed according to Eq.18.

$$
E_{k}=-\frac{\lambda_{i}}{\sum_{j=1}^{n} \lambda_{i}} \log \left(\frac{\lambda_{i}}{\sum_{j=1}^{n} \lambda_{i}}\right)
$$

Due to the noise effect on the signal decomposition performance of LMD, before do LMD for the signal, we use SVD to the signal to suppress the noise. The increment of singular entropy choose conservative strategy, then LMD decomposition add to signal to get PFs, and SVD denoising do again for PFs, after denoising the PFs sum to a new signal and do LMD again. Repeat above process until the decomposition of PF instantaneous amplitude and instantaneous frequency components to meet the high precision requirement of the time frequency analysis.

\section{SIMULATION AND TEST DATA PROCESS}

To verify the validity of the proposed method in this paper, using simulation signal and telemetry vibration signal in a test are processed to verify. Simulation signal using the sum of three different center frequencies of sinusoidal signal, at the same time adds Gauss white noise and $\mathrm{SNR}=3.5 \mathrm{~dB}$. The sample frequency is $500 \mathrm{~Hz}$, where $f_{1}=10 \mathrm{~Hz}$, $f_{2}=30 H z, f_{3}=80 H z$.

$$
x(t)=\sin \left(2 \pi f_{1} t\right)+\sin \left(2 \pi f_{2} t\right)+\sin \left(2 \pi f_{3} t\right)+n(t)
$$

The waveform of simulation signal can be shown as Fig.2. The result of LMD directly can be shown as Fig.3, and the decomposition result of LMD combined SVD denoising is shown as Fig.4. We can see that the proposed in this work has better performance.

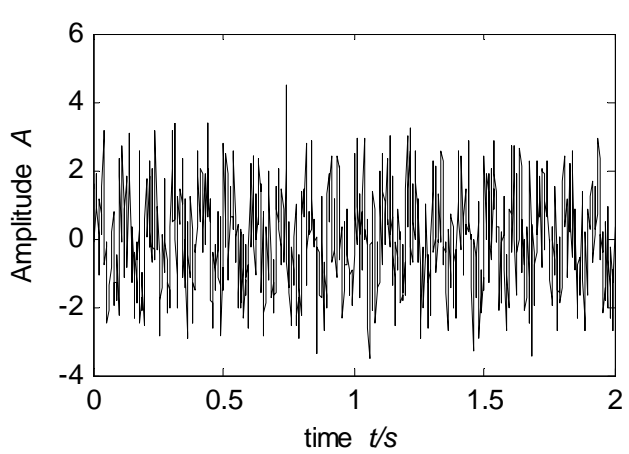

Figure 1. Waveform of simulation signal.
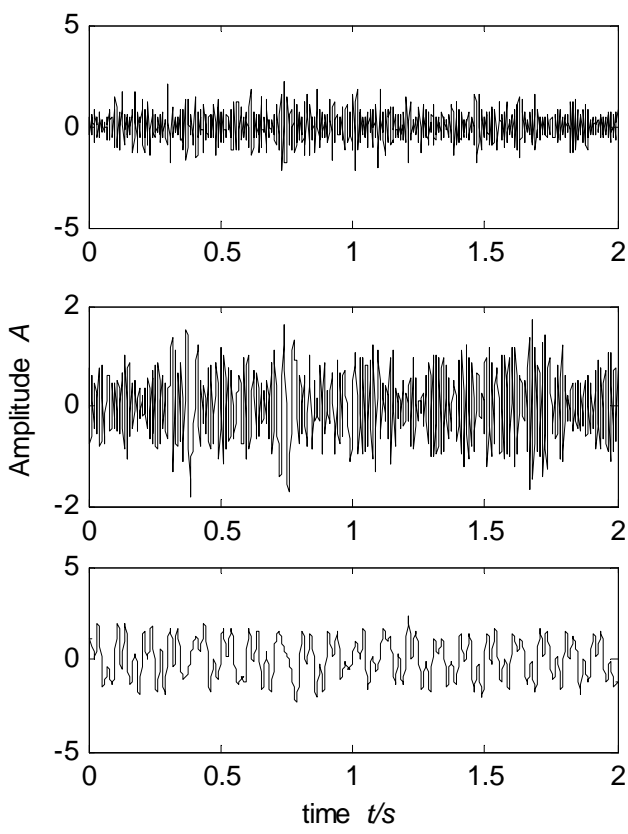

Figure 2. PFs of LMD. 

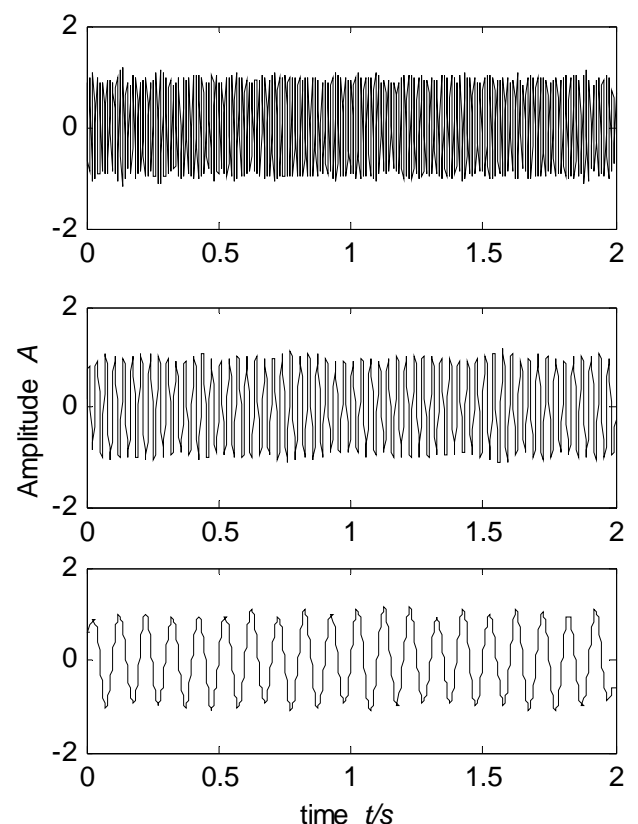

Figure 3. PFs of LMD combined with SVD

Test data of telemetry vibration signal sample frequency is $5 \mathrm{kHz}$ and waveform is shown as Fig.4. The result of LMD directly can be shown as Fig.3, and the decomposition result of LMD combined SVD denoising is shown as Fig.4.

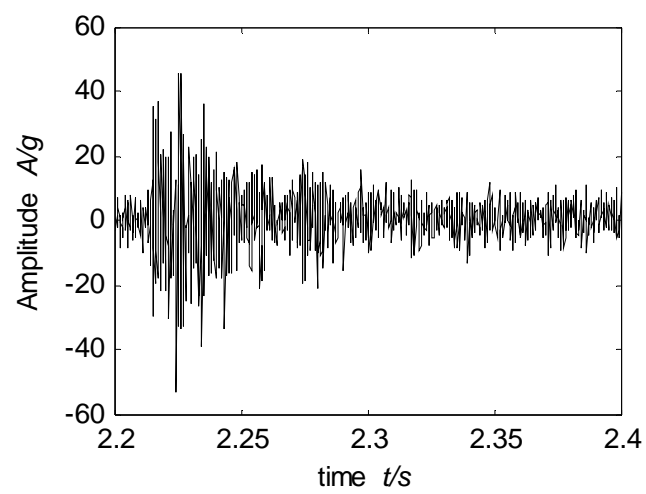

Figure 4. Waveform of test signal.
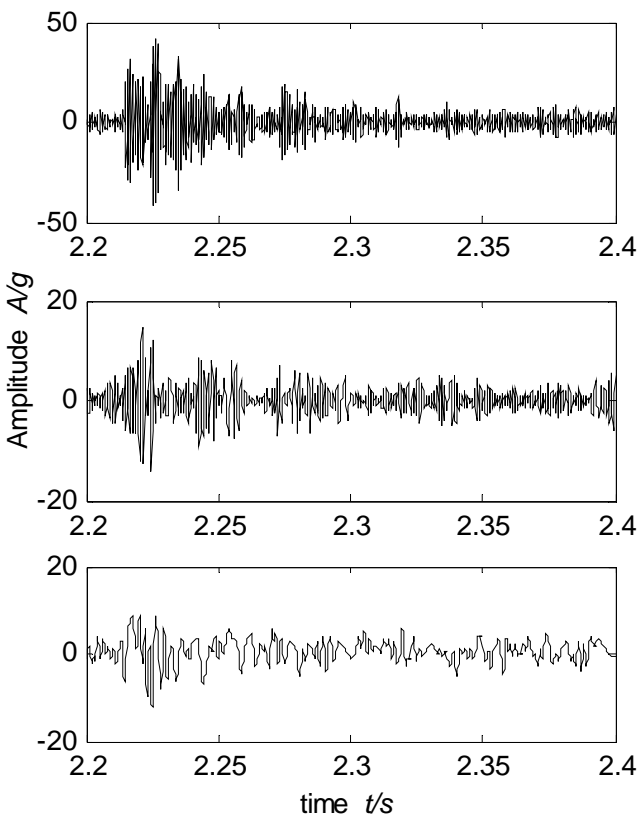

Figure 5. PFs of LMD.

\section{CONCLUSION}

LMD as a non-stationary signal analysis technology is a new method in the practical engineering, it has potential application value engineering, according to the actual acquisition signal with noise interference, SVD and LMD methods are combined, the original signal using SVD denoising, and the SVD denoising also embedded into the LMD decomposition process, effective suppress the noise on the LMD decomposition component instantaneous amplitude and instantaneous frequency effects, under the condition of low SNR still can obtain good performance of decomposition, so the method proposed in this work has certain project application value. 

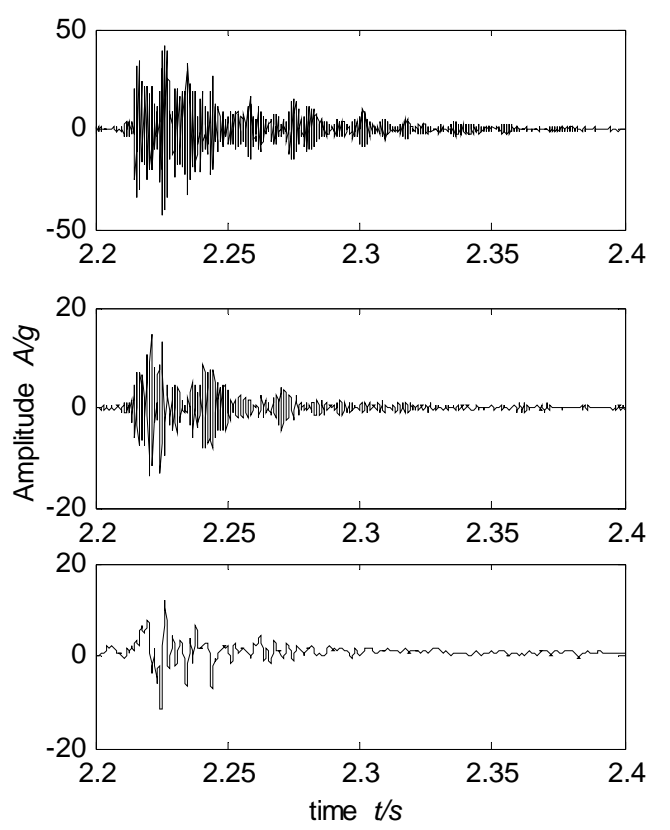

Figure 6. PFs of LMD combined with SVD

\section{REFERENCES}

[1] Wang Y X, He Zj, Zi Y Y. “A demodulation method based on local mean decomposition and its application in rub-impact fault diagnosis". Measurement Science \& Technology, vol.20, no.2, pp. 205- 209.

[2] Huan N E, Wu Z H, Long S R, et al. "On instantaneous frequency”. Advance in Adaptive Data Analysis, vol.1, no.2, pp.177-229, 2009.

[3] Wang Yanxue, He Zhengjia, Zi Yanyang, et al. "Several key issues of local decomposition method used in mechanical fault diagnosis”.Journal of Vibration and Shock, vol.31, no.9, pp.9-12,2012.

[4] Ren Daqian, Yang Shixi, Wu Zhaotong, et al. "Instantaneous frequency extraction method and experiment based LMD”. Journal of Zhejiang University (Engineering Science), vol.43, no.3, pp.523-528, 2009.

[5] Akritas A G, Malaschonok G I. "Application of Sigular Value Decomposition(SVD)". Mathematics and Computers in Simulation, vol.67, no.1, pp.15-31, 2004.

[6] Wu Chun-Guo, Liang Yan-Chun, Sun Yangfeng, et al. "On the equivalence of SVD and PCA”. Chinese Journal of Computer, vol. 27, no.2, pp.286-288, 2004.

[7] Zhao Lifeng, Yao Yuling. "Signal reconstruction by the SVD method”. Journal of Ocean University of Qingdao, vol.29, no.1, pp.101-106, 1999.

[8] Wang Taiyong, Wang Zhengying, Xu Yonggang, et al. "Empirical mode decomposition and its engineering applications based on SVD denoising”. Journal of Vibration and Shock, vol.24, no.3, 96-98, 2005. 